\title{
GÉNESIS DE LA FEDERAL TRADE COMMISSION Y BREVE ANALISIS DE LOS OBJETIVOS DEL DERECHO ANTITRUST NORTEAMERICANO Y EUROPEO
}

\author{
María Jeleztcheva Jeleztcheva*
}

\begin{abstract}
Sumario: 1. Introducción. 2.Génesis de los órganos antitrúst en los EEUU. Las comisiones de los estados y el génesis de la Interstate Commerce Commission. 3.Creación de la Federal Trade Commission y de la regulación antitrúst. 4. Los objetivos del derecho antitrúst y de los órganos antimonopolio estadounidenses. 5. Los objetivos del derecho de defensa de la competencia europeo y de la Comisión como órgano de defensa de la competencia en Europa.

In fact, that may occur in the long term, a convergence between the
\end{abstract}

- Resumen: La presente investigación tiene como objetivo abordar las metas actuales de las autoridades de los Estados Unidos y Europa, tratando de luchar contra las prácticas contrarias a la competencia, mediante la creación de estas autoridades, con énfasis en las diversas condiciones económica, social y política en ambos lados del Atlántico. Con relación a los objetivos de la legislación europea antimonopolio y de la Comisión Europea en la aplicación de la política de competencia debe recordar los objetivos de los Tratados en que la idea principal es la creación e integración del Mercado Único. Este objetivo no excluye la otra clase de los derechos económicos, sociales y políticos. De hecho, hay también que pueden ocurrir, en el largo plazo, una convergencia entre los objetivos de la legislación europea y los objetivos de la normativa de competencia de los Estados Unidos, mediante el establecimiento de una jerarquía de las metas en las áreas de la globalización económica y el posible establecimiento de una regulación global de la protección de la competencia

- Palabras-clave: defensa de la competencia; regulación; Estados Unidos y Europa.

- Abstract: This study deals with the current objectives of United States and European authorities, seeking to combat anticompetitive practices through the creation of these authorities, with emphasis on different economic, social and political on both sides of the Atlantic. With relation to the objectives of European antitrust law and the European Commission in the implementation of politics competition,the objectives of the Treaties should be reminded, which the main idea is the creation and integration of the Single Market. This objective does not exclude the other economic, social and political kinds. objectives of European law and the purposes of U.S. antitrust law by establishing a hierarchy of goals in areas of economic globalization and the possible establishment of a regulatory global antitrust.

- Keywords: competition defense; regulation; United States and Europe.

\section{Introducción.}

La creación de los primeros órganos de defensa de la competencia empezó en los Estados Unidos ${ }^{1}$ con el establecimiento de las independent commissions de los estados federados que fueron al principio unas comisiones parlamentarias ad

\footnotetext{
*Doctora en DerechoProfesora de la Universidad Carlos III de Madrid

${ }^{1}$ Se puede consultar mi Tesis Doctoral titulada Las autoridades de de defensa de la competencia: los EEUU, la UE, España y Bulgaria, estudio comparativo que se puede ver en http//:eprints.ucm.es/8185.
} 
hoc creadas para regular el sector del ferrocarril2: los problemas sociales que surgieron por los precios elevados del ferrocarril y la corrupción política fueron las principales razones de la creación de estas instituciones.

Una buena introducción a la situación político- económica después de la Guerra Civil de los EE.UU. se puede encontrar en AREEDA\&KAPLOW ${ }^{3}$ : en aquellos tiempos se podía observar una rápida expansión económica, cuyas principales causas han sido la población de los enormes territorios vacíos del Oeste, la construcción de ferrocarriles y nuevas fábricas ${ }^{4}$ que tenían que responder a las necesidades de los granjeros.Como es sabido, el crecimiento rápido de la economía en la mayoría de los casos es seguido por depresiones periódicas, excesos de producción y cambios rápidos en la estructura del mercado.

El descontento de la población por las altas tarifas ferroviarias y los precios altos a los que los fabricantes vendían el equipamiento para las granjas llevó a una presión constante sobre los poderes públicos ${ }^{5}$.Surgieron organizaciones como National Grange cuyo objetivo era defender los intereses de los farmers ${ }^{6}$.

En la situación descrita era lógico que los poderes públicos reaccionaran a los cambios creando normas e instituciones y estas instituciones- las comisiones independientes- surgieron en principio a nivel estatal.

\section{Génesis de los órganos antitrúst en los EEUU.Las comisiones de los estados y el génesis de la Interstate Commerce Commission.}

Las funciones de las comisiones estatales se limitaban a obtención de información, supervisión de la seguridad de los ferrocarriles y emsión de informes ${ }^{7}$.La explicación de la actividad limitada de estos órganos se puede

\footnotetext{
${ }^{3}$ AREEDA \&KAPLOW Antitrust Analisis: Problems, Text, Cases, 4a ed., ed.Little, Brown \& Co., 1988, pp.4850;ver asimismo PARZITS, K. A Primer to Antitrust Law and Regulatory Policy, ed.University Press of America,Lanham, 1987, p.30;SHENEFIELD, J. y STELZER,I. The Antitrust Laws: a Primer, op.cit., p.10;NAVARROSUAY,MadelCamen "Lapromulgación delaShermanAct:factoreshistóricos, económicosylegislativos", Revista de derecho mercantil N 253, julio-septiembre 2004.

${ }^{4}$ Sobre estetemaverKNITNER, E. The Legislation History of theFederal Antitrust LawsandRelatedStatutes, New York, 1978, p.11; LETWIN, W. "Conress and the Sherman Antitrust Law:1887-1890", U. Chi. L. Rev. 23, 1956. ${ }^{5}$ En palabras de BEVIS, H. "Procedure in the Interstate Commerce Commission- a Study in Administrative Law", 1 U. Cin. L. Rev.241(1927), las mutaciones industriales, financieras, geográficas y sociales han encargado el gobierno con una cantidad de deberes impropios.Así el mismo se veía obligado decidir sobre problemas, que hasta aquel entonces no habian surgido con tal intensidad en ninguna otra parte del mundo y tenía que reaccionar con medidas adecuadas e innovativas.

${ }^{6}$ Para la influencia del movimiento agrario sobre la aprobación de las leyes antitrrust estatales se puede leer el artículo "Antitrust before the Sherman Act", BOUDREAUX, D., DILORENZO, T.y PARKER, St. en McCHESNEY, F.\& SHUGART III, W. The Causes and Consequences of Antitrust, ed.Univ. of Chicago Press, 1995, pp.255- 270 , asimismo KOVACIC, W. "Public Choice and Origins of Antitrust" en la misma obra, p.250; KNITNER, E. "The Legislation History of the Federal Antitrust Laws and Releted Statutes", op.cit., p.7.

${ }^{7}$ MORENOMOLINA, A. M.,Laadministración por agenciasenlos Estados Unidos de Norteamérica, ed. Universidad Carlos III: Boletín Oficial del Estado, Madrid, 1995, p. 103.
} 
encontrar en la creencia en las fuerzas del mercado y su buen funcionamiento por sí mismo.

La siguiente fase de establecimiento de instituciones fue la creación de comisiones parlamentarias permanentes con las siguientes características:

- elección de los miembros por el Gobernador con la aprobación del Parlamento,

- mandato de sus miembros entre 2 y 6 años; la renovación de los mandatos se hacía por partes,

- en algunos casos se exigía que estuviesen compuestas por representantes de los partidos políticos existentes para evitar que la Comisión estuviera controlada por el partido al que pertenecía el Gobernador,

- las comisiones no respondían ante el Gobernador sino ante el Parlamento del estado federado (porque se consideraban agentes del órgano legislativo $)^{9}$.

Las elevadas tarifas ferroviarias, la discriminación y la necesidad de establecer restricciones sobre las prácticas abusivas de las compañías en el sector a nivel federal llevaron a la intervención del Congreso y a la creación en 1887, (después del detallado informe del Cullom Committee), de la Interstate Commerce Commission ${ }^{10}$ (Interstate Commission Act, 24 Stat. 379) que fue el modelo de la posterior Federal Trade Commission. Su régimen era parecido al de las comisiones de los estados federados:

- estaba compuesta por 5 comisarios de los cuales no más de 3 podían pertenecer a un mismo partido político, nombrados por el Presidente con el visto bueno del Senado,

- su mandato era de 5 años,

- causas tasadas de cese por el Presidente (ineficiencia, abandono del servicio o conducta delictiva en el ejercicio del cargo ${ }^{11}$ - Sec. 13 Interstate Commission Act, 25 Stat. 379),

- la Interstate Commerce Commission respondía ante el Parlamento.

Se trataba de una agencia creada por el Parlamento, que le otorgaba las competencias para cumplir sus funciones dentro del Poder Ejecutivo pero al margen de la organización administrativa dependiente del Presidente ${ }^{12}$. En la primera regulación de la Interstate Commerce Commission se preveía la

\footnotetext{
${ }^{8}$ Hoy las manifestaciones de la misma convicción se pueden leer en las obras de los economistas y juristas de Chicago y Post- Chicago.Sobre la aceptación de la competencia como causa principal del desarrollo económico de los EE.UU vease ANDERSON, S. "Federal Trade Commission - What Is It and What Does It Do?" 4 S. D. L. Rev. 117(1959).

${ }^{9}$ SALVADOR MARTÍNEZ, M., Las Autoridades Independientes, ed. Ariel, Barcelona, 2002, p. 107.

${ }^{10}$ GELLHORN, E. Antitrust Law and Economics in a Nutshell, ed. West Publishing Co., St. Paul, Minn., 1981, pp.16-18;el artículo de BEVIS, H. "Procedure...", op.cit.

${ }^{11}$ SALVADOR MARTÍNEZ, M., Las Autoridades Independientes", op.cit, p. 110.

${ }^{12}$ PIERCE, R, SHAPIRO, S. y VERCUIL, P. Administrative Law and ProceS “,ed. Little, Brown \& Co., New York, 1999, p. 29.
} 
posibilidad de supervisión y control por parte del Departament of Interior que era el encargado de aprobar sus gastos, recibir informes de la Interstate Commerce Commission y darles traslado al Parlamento. Más tarde se introdujeron cambios para evitar cualquier vía de influencia del Presidente sobre la Interstate Commerce Commission y los informes se enviaban directamente al Congreso.De este modo se creó un órgano, que tenía cierta independencia respecto al Presidente y actuaba en amplia discrecionalidad, contando con la ventaja de un procedimiento administrativo más flexible que el proceso judicial para poder realizar las importantes tareas de regulacion del comercio.

La Interstate Commerce Commission estaba facultada para recibir quejas, investigarlas y, en su caso, acudir a los tribunales, mantenía informado al Gobierno y proponía las normas necesarias al Parlamento.En principio, las órdenes dictadas por la ICC no tenían efecto vinculante y los juzgados podían emplear las observaciones de la agencia solo en relación con un determinado juicio. Pero sus competencias se fueron ampliando, paralelamente a la evolución del mercado estadounidense, ya que se le atribuyeron competencias ejecutivas ${ }^{13} \mathrm{y}$ cuasijudiciales ${ }^{14}$. Además la Interstate Commerce Commission decidía la política pública a seguir en el comercio. Con la Hepburn Ammedment de 1906 la Interstate Commerce Commission fue dotada con la potestad de fijar tarifas ${ }^{15}$, que se considera por parte de la doctrina norteamericana una potestad cuasi legislativa. Más tarde, en 1910 con la Ley Mann-Elkins (36 Stat. 539) se creó un tribunal especial en materia de comercio $^{16}$.

Se puede resumir que, en un ambiente político y económico, caracterizado con rápidas transformaciones, era imprescindible crear un órgano que pueda responder a estos cambios y, tratándose de una medida innovativa, el Congreso ha

${ }^{13}$ Ver BEVIS, H. "Procedure...", op.cit.; SALVADOR MARTÍNEZ, M.Las Autoridades Independientes, op.cit, p.93;las facultades de la ICC en los años después de su creación están analizadas detalladamente por RALSTON, J. "The Intersate Commerce Commission", 12 Am. Law 297(1904); sobre la atribución de poderes ejecutivos, legislativos y judiciales a la ICC ver asimismo SCHVARTZ, B. Administrative Law, ed. Little, Brown \&Co., Toronto, 1991, pp.28-29.

${ }^{14}$ Como señala Bemstein, M., cit. por Moreno Molina, Ángel Manuel, “La administración por agencias en los Estados Unidos de Norteamérica “, ed. Universidad Carlos III: Boletín Oficial del Estado, Madrid, 1995, p. 109: "la razón principal para la creación de las Comisiones Independientes ha sido la restricción de ciertas prácticas abusivas de las empresas privadas. El aparato administrativo empleado por las Comisiones se empleó en la consideración casuística de problemas y asuntos separados, de un modo que se aproximaba muy de cerca al comportamiento de un juez y de un jurado... la regulación se forjó sobre un molde judicial y los comisarios se consideraban como jueces administrativos" - Bernstein, M. "Testimony before the Antitrust Subcommitee of The House Judiciary Commission of Monopoly Problems in regulated industries", 1956. "Les podía exigir información, investigar, prohibir, resolver, aplicar, impetrar el auxilio judicial, imponer multas, etc..." - Moreno Molina, Ángel Manuel, "La administración por agenciasen los Estados Unidos de Norteamérica", ed. Univ. Carlos III: Boletín Oficial del Estado, Madrid, 1995, p. 106.

${ }^{15}$ En este contexto- Bikle, H. "Power of the ICC to Prescribe Minimum Rates", 36 Harv. Law Rev.5 (1922-1923). 
ampliado las facultades de este órgano paulatinamente, respondiendo a las crecientes necesidades de un equilibrio político -económico.Se creó la primera agencia reguladora independiente, que se caracterizaba principalmente con el "aislamiento" de los tres poderes públicos, quedando en el esquema del Ejecutivo - un fenómeno que se puede observar asimismo en el caso de la posterior Federal Trade Commission(FTC).Además, se le atribuyeron una serie de facultades, propias de los demás poderes públicos y estas facultades se han venido ampliando poco a poco , como se va a observar asimismo en el caso de la FTC.

De este modo, como alternativa de la regulación de la competencia por parte del mismo mercado se introdujo un control público sobre la competencia.

Hasta aquí se han expuesto en breve las características más importantes de la Interstate Commerce Commission como predecesora de la Federal Trade Comisión, para poder analizar después los objetivos de la política de competencia, promovida por este órgano.

\section{Creación de la Federal Trade Commission y de la regulación antitrúst.}

La Federal Trade Commission es la primera institución de defensa de la competencia.

Como señalan BREYER y STEWART "el principio de mercados libres e iguales, no restringidos por el poder público... fue un principio fundamental en la América post-revolucionaria". ${ }^{17}$

A pesar de esto a finales del s. XIX y principios del s. XX (como se ha expuesto anteriormente) surge la necesidad de la primera regulación de los mercados y esta intervención de los poderes públicos es cada vez más eficaz por la necesidad de establecer un control efectivo sobre el creciente poder de los trusts.La expansión industrial llevó a la creación de una enorme cantidad de agentes de mercado y algunas de estas empresas llegaron rápidamente a

${ }^{16}$ Moreno Molina, A. M., “La administración por agenciasen los Estados Unidos de Norteamérica”, op. cit., p. 111. ${ }^{17}$ BREYER, St. y STEWART, R. Administrative Law and Regulatory Policy, $3^{a}$ Edición, ed. Little Brown and Co., Boston, 1992, p.143;en esta época se han expresado en favor de la laissez faire varios economistas contemporáneos: la tesis más citada es de J. B. CLARK(1887), cit. por DEMZETZ, H. La competencia:aspectos económicos,jurídicos y políticos, Alianza Editorial, Madrid, 1986, p.25 y por NEALE, A. The Antitrust Laws of United States..., op.cit., p.13, segun la cual "Las asociaciones tienen sus raíces en la naturaleza de la industria social y son normales en su origen, desarrollo y funcionamiento práctico.No deben ser desalentadas por los científicos ni suprimidos por los legisladores.Son el resultado de una evolución y son el feliz resultado de una competencia tan normal, que su supresión hubiera significado una amplia ruina.Un intento de suprimirlas con éxito por ley implicaría la reversión de los sistemas industriales hacia otros ya deshechos y la renovación de los abusos de los que la sociedad ha escapado en su marcha hacia el desarrollo."Estas visiones, que recibieron el nombre de "Darvinismo social" estaban muy extendidas entre los economistas de la época de la creación de las normas e instituciones antitrust, pero no ejercieron influencia sobre los legisladores y esta puede ser una buena perspectiva para considerar que en los orígenes del "movimiento antitrust" las razones de eficiencia económica y del bienestar del consumidor no eran 
dimensiones importante ${ }^{18}$ creando desventajas para sus competidores.A esto se añadía la así llamada "ola de fusiones" ${ }^{19}$ que era la manifestación de una consolidación corporativa ${ }^{20}$, con efectos importantes para las próximas décadas.Por ejemplo, existían los así llamados "money trusts", que se creaban en base a la consolidación de varias industrias y los directivos de las empresas que formaban parte de estos trusts eran miembros de varios consejos directivos de otras empresas.Así se creaban interconexiones entre las industrias ${ }^{21}$ y esto se podía ver como una amenaza del estado democrático norteamericano y los derechos individuales. El Congreso, después de establecer una regulación efectiva de los abusos en el sector del ferrocarril, volvió al problema del control monopolístico sobre la industria y el comercio. Con este objetivo se aprobaron la Sherman Antitrust Act ${ }^{22}$ (1890) y la Clayton $A_{c t}{ }^{23}$ (1914). Hasta la aprobación de la Federal Trade Commission

las dominantes.Hoy día existen opiniones en contra de la regulación pro-competencia y una gran parte de estas doctrinas están basadas en la idea que el progreso económico y las innovaciones se pueden realizar en industrias altamente concentradas: ver SHUMPETER, J. Capitalismo, Socialismo and Democracia, ed. Folio, Barcelona, 1996, según quien la innovación continua lleva a tecnologías para producir productos sustitutivos y esto es posible exclusivamente en base a la existencia de monopolios estables; ver asimismo GALBRAITH, J. K. Capitalismo Norteamericano,

ed.Agora, Buenos Aires, 1955 y MACLAURIN, W. Invention and Innovation in the Radio Industry,ed. Macmillan, New York, 1949.Una gran parte de los representantes de la Escuela de Chicago tampoco aprueban la mayonía de las medidas antitrust, ver por ejemplo STIGLER, G. The Economic Effects of the Antitrust Laws en MCCHESNEY,F. y SHUGART III, W. The Causes and Consecuences of Antitrust:the Public-Choice Perspective, ed.Univ. of Chicago,Chicago,1995.

${ }^{18}$ Algunos, como el Juez Brandeis negaban la posibilidad de las grandes empresas de ser eficientes y creían que la política antitrust tiene que promover la pequeña empresa.

${ }^{19}$ Sobre este fenómeno ver WINERMANN "The Origins of the FTC: Concentration, Cooperation, control and Competition" 71 Antitrust Law Joumal 2003, pp.6-7; AREEDA\&KAPLOW Antitrust Analisis..., op.cit.,pp.49-50, NAVARRO SUAY, Ma del Carmen "La promulgación de la Sherman Act: factores históricos, económicos y legislativos", Revista de derecho mercantil N 253, julio-sept. 2004.

${ }^{20} \mathrm{Se}$ dan los siguientes ejemplos:72 consolidaciones llevaron a la creación de empresas que controlaban $40 \%$ de la industria, otras 42 consolidaciones llevaron a un control de más de 70\% (LAMOREUX, N., cit. por WINERMANN, M., "The origins of the FTC", op.cit., p.7). Además, 12\% de las empresas del país empleaba $75 \%$ de los trabajadores, representando $80 \%$ de la producción industrial total del paísBARACK, O., cit. por LANG, J. "Legislative History of the FTC Act", 13 W. L. Rev.6, 1974.

${ }^{21}$ En este sentido ver BRANDEIS, L. Other People's Money and how the Bankers Use It (1913).

${ }^{22} \mathrm{El}$ senador John Sherman fue hermano de un famoso financiero y empresario que durante muchos años ha expresado su descontento por los abusos que se producían en el comercio. Así Sherman ha introducido tres anteproyectos de la Ley con el fin de destruir las concentraciones del capital que según su opinión rompían el equilibrio en la economía norteamericana. De este modo las pequeñas empresas eran incapaces de competir con el leviathan industrial (MILLER, R. y JENTZ,G. Business Law Today, ed.ITP, NY, 2000, p. 813).

${ }^{23}$ En 1914 el Congreso ha optado por reforzar el derecho antitrust por medio de esta Ley. Así, la Clayton Act incluía prácticas específicas no previstas en la Sherman Act. Las disposiciones más importantes de Clayton Act incluyen 4 diferentes formas de comportamiento en el comercio, que han sido declaradas ilegales pero no criminales. Los 4 supuestos estaban condicionados por la posibilidad de dañar sustancialmente la competencia y por la posibilidad de crear poder monopolístico (MILLER, R. y JENTZ,G. Business Law Today, ed.ITP , 2000, p. 813);GELLHORN, E .Antitrust Law and Economics in a Nutshell, op.cit., pp.25-30. 
Act (1914) $)^{24}$ el Departamento de Justicia estaba encargado de la aplicación de la Sherman Act (tanto cuando se trataba de violaciones criminales como civiles). Puesto que las infracciones de la Clayton Act no han sido cualificadas como crímenes, el Departament of Justice podía aplicar dicha ley solamente en procedimientos civiles. Así el Departament of Justice podía exigir de los jueces imponer la sanción "divestiture" (hacer que la empresa responsable deje de ejercer una o más de sus funciones como empresa) o la disolución de la misma. De este modo, hasta la aprobación de la Federal Trade Commission Act las leyes Sherman y Clayton se aplicaban por los tribunales comunes, iniciándose los procedimientos por el Departamento de Justicia.

Se puede afirmar que la idea de la creación de la Federal Trade Comisión surgió durante el mandato del Presidente T. Roosvelt que fue elegido el año $1901^{25}$.El Presidente era consciente de que hay que distinguir las combinaciones industriales que contribuyen a la eficiencia productiva de las que eliminan a sus competidores y que el control adecuado sobre los abusos de estos acuerdos o cooperaciones se puede realizar adecuadamene por medio de una comisión administrativa ${ }^{26}$. En 1903, creó el Bureau of Corporations ${ }^{27}$ que ha publicado estudios importantes sobre organizaciones como Standard Oil Co. o American Tobacco Co.Así, durante este período han surgido problemas importantes en relación con la necesidad de determinar en qué caso y cómo, de acuerdo con las exigencias del desarrollo de la industria y el comercio, de la igualdad de oportunidades y competividad internacional, se puede controlar el crecimiento corporativo.La idea de Roosevelt era racionalizar la economía, asesorar su funcionamiento, no simplemente disolver los trusts. Durante el mandato del Presidente W. Wilson se presentó un paquete de propuestas legislativas con el objetivo de institucionalizar el Bureau of Corporations: se trataba de una agencia creada no sólo con el propósito de asesorar a los juzgados, sino para establecer

\footnotetext{
${ }^{24}$ La Federal Trade Commission Act fue aprobada por 2 objetivos:-Esta ley se extendía sobre todos los comportamientos anticompetitivos, incluidas también las conductas que no infringían las normas de las Shermano Clayton Act (Sec. 5laFederal TradeComisión Act).-Secreabala Federal TradeCommission para aplicar la Sec. 5 de la Federal Trade Commission Act y también la Clayton Act (MILLER, R. y JENTZ,G Business Law Today, ed.ITP, 2000, p. 813).

${ }^{25}$ Es famosa la frase de Roosevelt "La libertad en la democracia no es garantizada cuando la gente tolera el crecimiento del poder privado hasta tal punto que ya es más fuerte que el propio estado democrático"(1938), cit. por Morgan,T .Modern Antitrust Law and its Origins, ed.West Publishing Co., St. Paul, Minn., 1994, p.113.

${ }^{26}$ ROOSEVELT, T. "Confession of Faith", 6 de agosto de 1912 en 17 Works of Theodore Roosevelt; sobre el período en el principio del siglo XX en relación con la institucionalización del antitrust ver THORELLI, H.TheFederalAntitrustPolicy.Origination ofanAmerican Tradition, ed.S.N.,Stockholm, 1954,pp.558-561 ylaobradeLETWIN, W.Law and Economic Policy in America: the Evolution of the Sherman Antitrust Act, ed.Edinburg U.P., 1967. ${ }^{27}$ WINERMAN, M. "The Origins of the FTC: Concentration, Cooperation, control and Competition" 71 Antitrust Law Journal 2003, pp.3-97;McCRAW, T. Prophets of Regulation, ed.Belknap, Cambridge, Mass., 1984, pp.81-142, THORELLI, H. The Federal Antitrust..., op. cit., p.411 - 421;KNITNER, E. The Legislative History..., vol. II, op. cit., p.994.
} 
sus propios criterios respecto a las prácticas anticompetitivas ${ }^{28}$. Con la aprobación de la Federal Trade Commission Act se perseguía el fin de permitir la aplicabilidad de la normativa antitrust por un órgano administrativo debido al descontento generado por las interpretaciones judiciales excesivamente restrictivas ${ }^{29}$ y a esto se añadía la debilidad y parcialidad del Departamento de Justicia en su política de investigaciones y sanciones, que acabó en una inseguridad jurídica ${ }^{30}$.En la doctrina norteamericana existe la convicción que la creación de la FTC es consecuencia de la sentencia del Tribunal Supremo de los EE. UU. Standart Oil Co. of New Jersey v. U.S., 221 U.S.1 (1911) ${ }^{31}$, que por primera vez establece la rule of reason en el derecho antitrust norteamericano, y esta convicción está basada en las preocupaciones en aquella época de que los juzgados serán los "nuevos legisladores" y que interpretarán la ley o bien de una manera más amplia(en interés de los trusts) o bien de una manera más estrecha, creando obstáculos para el comercio y los acuerdos entre los fabricantes ${ }^{32}$. De este modo el proyecto de creación de una agencia administrativa recibió apoyo de tres grupos de intereses diferentes:

- en primer lugar de los que estaban en contra del "Big Business" y esperaban una restauración del sistema competitivo antes existente; ellos estaban a favor asimismo a la idea de crear normas más detalladas y precisas,

- la gente del mundo de los negocios estaba a favor de la creación de tal órgano administrativo por diferentes razones: por la necesidad de supervisión de las grandes corporaciones para sancionar los abusos; por motivos de seguridad

\footnotetext{
${ }^{28}$ Sería interesante estudiar los debates en el Congreso con motivo la aprobación de la Federal Trade Commission Act en el contexto socio-político de los EEUU de aquel entonces: según NEALE, A. D.(op. cit., pp.178-180) la creación de la FTC se puede ver asimismo como una consecuencia del compromiso de los que defendían la "lista negra" de conductas que se deberían incluir en la Ley y los que propugnaban la inclusión de fórmulas abiertas que se podrían concretar por un órgano especializado en materia de las prácticas anticompetitivas; ver asimismo WINERMAN, M. The Origins of the FTC, op. cit., pp.4-5, donde se analizan las tres posturas contradictorias , expresadas en el Congreso: de F. Newlands, según quien había que autorizar la Comisión para aplicar una normativa general, de Hollis,H. que defendía la postura que había que establecer definiciones más precisas a seguir por este órgano y de Cummins, A., para quien la Sec. 5 de la FTC Act era solo un instrumento, pero no el medio más importante para que el Congreso dirija la política antitrust.Existe asimismo diferencia entre los objetivos, que los tres diputados ven como prioridades de esta nueva institucion; se puede leer un comentario detallado sobre este tema asimismo en Federal Trade Commission $75^{\text {th }}$ Aniversary Simposium, 58 Antitrust L. J. 797, 1989.

${ }^{29}$ MARTÍN-LABORDA, Antonio Robles, Libre competencia y competencia desleal, ed. La Ley, Madrid, 2001, p. 146;

${ }^{30}$ MORENO MOLINA, A. M. Aspectos institucionales de la defensa de la competencia, en Derecho Europeo de la Competencia, CALVO CARAVACA, Alfonso Luis y MORALES LIMONES, Pilar Blanco, ed. Constitución y Leyes, S. A., Madrid, 2000, p. 89.

${ }^{31}$ Más tarde volveré sobre el tema del significado de la rule of reason para el derecho antitrust norteamericano con propósito de explicar el proceso decisorio de la FTC.

${ }^{32}$ WINERMAN, M. "The Origins of the FTC: Concentration, Cooperation, Control and Competition" 71 Antitrust Law Journal 2003, pp.3-97; asimismo AREEDA\&KAPLOW Antitrust Análisis:..., op.cit., p.61.
} 
jurídica: se tenía que saber qué actos eran ilegales y cuales eran lícitos y esto podía determinarlo con más exactitud un órgano administrativo; según estas opiniones la comisión no debía tener amplios poderes de regulación y publicidad,

- en tercer lugar algunos propugnaban la posibilidad de conceder licencias a las grandes corporaciones interestatales e incluir su régimen en el Federal Law y esto exigía la creación de una agencia gubernamental para autorizar $^{33}$ estas corporaciones.

A esto se añadía que los tres partídos políticos(Demócratas, Republicanos y Progresistas) estaban prácticamente a favor de la creación de tal órgano administrativo ${ }^{34}$.

De este modo las razones que llevaron a un apoyo público unánime en la primera década del siglo pasado, llevaron a la creación de la FTC. Considero importante hacer algunas notas sobre la intención del Congreso para crear las normas e instituciones antitrust, porque este "congressional intent", según la doctrina norteamericana, es la clave para la interpretación del derecho antitrust tanto por la FTC y la División Antitrust, como también por los juzgados.

En la opinión de DIRLAM\&KAHN ${ }^{35}$ se dan dos principales causas para la regulación antitrust (y de aquí del establecimiento de instituciones antitrust por el Congreso):

A.Los controles se introducen con objeto de adecuar la situación al sentido de equidad y justicia en la sociedad, sobre todo protegiendo los derechos de los consumidores ${ }^{36}$ y los demás competidores.Aquí no se trata solamente de un objetivo social, sino también económico, porque el crecimiento de la demanda lleva a la fabricación de mejores productos a precios más bajos.Una visión en este sentido ofrece Schwartz ${ }^{37}$, señalando que las ganancias económicas no pueden ser un factor exclusivo y decisivo cuando se resuelven las controversias basadas en las políticas antitrust: se afirma que el legislador ha buscado soluciones que aportan justicia y equidad.

B.Los controles buscan mantener el sistema de equilibrio(separación) de poderes ${ }^{38}$ (checks and balances) como base del funcionamiento del Estado

\footnotetext{
${ }^{33}$ CUSHMAN, R. The Independent Regulatory Commissions, ed. Octagon Books, New York, 1972, pp.178-206 ;asimismo Federal Trade Commission 75 ${ }^{\text {th }}$ Aniversary Simposium, 58 Antitrust L. J. 797, 1989.

${ }^{34}$ ANDERSON, S. "Federal Trade Commission - What is It and What Does it Do?",4 S. D. L. Rev.117, 1959.

${ }^{35}$ DIRLAM\&KAHN Fair Competition:The Law and Economics of Antitrust Policy, ed.Greenwood Press, Westport, Connecticut, 1954, p.16.

${ }^{36}$ Según ADAM SMITH "la consumición es el único objetivo de la producción", The Wealth of Nations, ed.Modern Library, Book IV, Chapter 8.

${ }^{37}$ Ver SCHWARTZ, L. Justice and other non-economic goals of antitrust, University of Pennsylvania Law Review, vol.127, N 4, abril 1979.

${ }^{38}$ Sobre estetemaverDRLAM\&KAHN Fair Competition the Lawand Economics of Antitrust Policy, ed. Greenwood Press, Newport, Connecticut, 1970, p.17 y en general BREYER\&STEWART Administrative Law, op.cit., p.33-66 y FOX, W. Understanding Administrative Law, 2ed., ed. Mattew Bender, New York, 1995, p.34.
} 
norteamericano y con objetivo de asistir(por medio de normas e instituciones) este sistema de balance de poderes.En este caso el objetivo del regulador es minimizar la coerción mediante una política antitrust que debe ser flexible.

Se ha de recordar que el concepto de separación de poderes describe un mecanismo específico, concediendo diferentes funciones a diferentes actores.Así según MADISON ${ }^{39}$ si el poder tiene por naturaleza la característica de expandirse debe ser efectivamente limitado al sobrepasar los límites correspondientes.Esto significaría controlar los tres poderes para asegurar una seguridad práctica de cada uno de ellos contra la invasión de los otros dos.

En el caso de las agencias independientes(como se va a apuntar más adelante en relación con la FTC), se trata de una forma de balance entre el Legislativo y el Ejecutivo, aunque garantizado por una concentración excesiva de funciones en un mismo órgano ${ }^{40}$.LAWSON señala que el Tribunal Supremo de los EE.UU. nunca ha cuestionado esta concentración de funciones en un mismo órgano en base a la doctrina tradicional de separación de poderes ${ }^{41}$.

Es preciso hacer referencia asimismo a la tesis de HYLTON ${ }^{42}$, segun cual la legislación antitrust es consecuencia de un compromiso de intereses.En esta dirección conducen asimismo las reflexiones de EKELUND, R. Jr., McDONALD,M. y TOLLISON, R. "Business Restraints and the Clayton Act of 1914: Public or Private Interest Legislation?".43

\section{Los objetivos del derecho antitrúst y de los órganos antimonopolio estadounidenses.}

La variedad de doctrinas actualmente existentes sobre los objetivos del derecho antitrust es cosecuencia de las diversas interpretaciones del "congressional intent" para la creación de las leyes e instituciones antitrust.Haré un breve resumen ${ }^{44}$ de estas doctrinas principalmente por dos razones: la primera razón es que cada una de ellas ofrece una perspectiva diferente para la interpretación de las normas antitrust, influyendo directamente sobre el proceso decisorio de la FTC(y asimismo de la División antitrust y los juzgados); la segunda razón es que algunas de estas doctrinas y más concretamente la que defiende la idea de la dispersión de poder como objetivo del derecho antitrust justifica la concesión de las diferentes facultades de la FTC que, desde los tiempos de su creación se han venido ampliando.

\footnotetext{
${ }^{39}$ MADISON, J. The Federalist No 48, ed. The Modern Library, New York, 1787, pp.321- 325.

${ }^{40}$ LAWSON, G. "The Rise and Rise of the Administrative State",107 Harv. L. Rev.,1231,1994.

${ }^{41}$ LAWSON, G. Federal Administrative Law, ed. West Publishing, St. Paul, Minn., 1998, pp.167- 168.

${ }^{42}$ HYLTON, K Antitrust Law:Economic Theory and Common Law Evolution", ed. CambridgeUniv., 2003, pp.38-39.

${ }^{43}$ En McCHESNEY, F. y SHUGART III, W.The Causes..., op.cit., p.273.

${ }^{44}$ Se puede consultar mi Tesis Doctoral titulada Las autoridades de de defensa de la competencia: los EEUU, la UE, España y Bulgaria, estudio comparativo que se puede ver en http//:eprints.ucm.es/8185.
} 
En primer lugar me voy a referir a la tesis de THORELLI, H.45, según la cual en el año 1890 la legislación antitrust ha sido la proyección de la filosofía de la libre competencia sobre la política pública, en sentido que si se eliminasen los obstáculos monopolísticos en la industria, la libre competencia empezaría a funcionar automáticamente ex definitione. Además, según THORELLI no ha existido un "congressional intent" unidireccional de conseguir una mayor eficiencia económica, sino que se han perseguido también otros objetivos de índole social.

Según otros autores, entre los cuales LANDE, $R^{46}$ y BORK, ${ }^{47}{ }^{47}$ la visión dominante en el Congreso en aquel entonces ha sido prevenir una "injusta" transferencia de beneficios de consumidores a productores.La finalidad de la política antitrust se acepta como protección del bienestar de los consumidores(precios más bajos y alta calidad de los productos).Este objetivo no se contrapone a la consecución de una mayor eficiencia económica. Sin embargo BORK considera que la protección del pequeño empresario no es objetivo de las leyes antitrust ${ }^{48}$.

En palabras de MILLON, D. la creación de enormes empresas de posición dominante en la industria se veía como una amenaza para las instituciones democráticas.Esta visión se relaciona con la idea de dispersión del poder como objetivo del derecho antitrust(y de la política de las instituciones antitrúst). - HOFSTADTER, $\mathrm{R}^{49}$.

FOX, E. ${ }^{50}$ afirma, que la intención del Congreso ha sido asegurar la posibilidad de que los individuos y las empresas compitan en una igualdad de oportunidades.

${ }^{45}$ Thorelli, H. "The Federal Antitrust Policy", ed. Nordest Forlag, Stokholm, 1954, p.227.

${ }^{46}$ LANDE, R. "Wealth Transfers as the Original and Primarly Concern of Antitrust: the Efficiency Interpretetion Challenged", 34 Hastings L. J.65, 1982.

${ }^{47}$ BORK, R. "Legislative Intent and the Policy of the Sherman Act", 9 J. L \& Economics7, 1966.

${ }^{48}$ Sobre todas estas cuestiones es interesante la obra de BORK, R. The Antitrust Paradox: the policy at war with itsel, Simon and Shuster, NY,1997.

${ }^{49}$ HOFSTADTER, R. "What Happened to Antitrust Movement?" en The Paranoid Style in American Politics and Other Essays, ed.Knopf, New York, 1965,pp.205-210.Según otros autores se trata de objetivos políticoeconómicos - "dispersar el poder económico" - SHENEFIELD, J. y STELZER, I.The Antitrust Laws: APrimer, ed. AEIWashington,1998y AMATO.G.Antitrustand theBounds of Power: theDilemma ofLiberalDemocracy in the History of Power, ed. Hart Publishing, Oxford, 1997, p.97; según FERNÁNDEZ ORDOÑEZ, M.A. " para los norteamericanos la competencia no es sólo un medio para conseguir que funcionen mejor los mercados, sino que es un fin en sí mismo" : se trata de la filosofía de evitar la acumulación de poder en unos pocos. "El objetivo es reducir el poder de algunos, aumentando la competencia e impedir el monopolio no sólo por razones de que la economía así funcionara mejor, sino para evitar una concentración de poder excesiva".No eran los efectos de la competencia económica lo que preocupaba más en los EEUU, puesto que, en ese mismo año de 1890, en que se aprobó la Ley Sherman, el Congreso aprobó también una ley proteccionista, la Ley McKinley, por la cual se elevaban sustancialmente los aranceles. Mejorar la eficiencia a través de la competencia interna, no era el principal objetivo que se buscaba con la legislación antimonopolios, sino impedir la concentración de poder. - FERNÁNDEZ ORDOÑEZ, M. A. La competencia, Alianza Editorial, Madrid, 2002, pp.20 21.Considero que para entender los objetivos y el funcionamiento de la FTC es importante cono cer las razones para la creación de las leyes antitrust y las instutuciones de defenca de la competencia y es conveniente aceptar la perspectiva de AMATO y FERNÁNDEZ ORDOÑEZ .

${ }^{50}$ FOX, E. Industrial Concentration and the Market Sistem: Legal, Economic, Social and Political Perspectives, ed.ABA, Chicago, 1979. 
En el derecho norteamericano se ha sostenido unánimamente que las políticas de defensa de la competencia y de defensa de los consumidores y usuarios tienen una finalidad, la maximización de la eficiencia que lleva a la maximización del bienestar de los consumidores y usuarios ${ }^{51}$. Esta teoría utilitarista fue aceptada en la actualidad por la Escuela de Chicago: la maximización de la eficiencia como único fin del derecho antitrust está aceptada unánimemente por la jurisprudencia estadounidense ${ }^{52}\left(\mathrm{POSNER}, \mathrm{R}^{53}\right)$.Hay que destacar que a partir de los años -70 el Tribunal Supremo de los EEUU emplea la perspectiva de la eficiencia económica en la mayoría de sus decisiones ${ }^{54}$.

Esbozando algunas de las doctrinas citadas, KOVACIC, W. ${ }^{55}$ ofrece asimismo una interpretación de este tema por la escuela de la Public Choice, según la cual el Congreso ha creado la FTC y otras instituciones reguladoras de la "Progressive Era" para servir a los intereses de los círculos de negocios.Se considera que la obra de KOLKO, G. "The Triumph of Conservatism"(1963) y su análisis de la creación de la FTC y ICC ha dado el origen a la "teoría de las capturas".

Una visión contemporánea ofrece MAY, J. ${ }^{56}$, que , analizando los objetivos señalados, llega a la conclusión que el Congreso no ha perseguido un único objetivo, creando leyes e instituciones antitrust y que su intención ha sido "multidimensional":la Sherman Act ha sido aprobada para restablecer el equilibrio y el orden social, político y económico existente antes de la industrialización y para suprimir la creación de grandes organizaciones industriales, que han amenazado con romper los pilares de este orden.En la tesis de MAY la mayoría de los objetivos del derecho antitrust que hoy se cree que están en conflicto, prácticamente se refuerzan mútuamente y estan subordinados a dicho objetivo.MAY y HOVENKAMP ${ }^{57}$ afirman que los creadores de las leyes e instituciones antitrust han perseguido todos los objetivos citados(prevenir la distribución injusta de la riqueza, la eficiencia económica,el bienestar del consumidor, ofrecer una igualdad de oportunidades, la dispersión del poder) a la vez, sin considerar que existen controversias entre ellos ${ }^{58}$ : se puede resumir que no existe objetivo dominante, sino una jerarquía de

\footnotetext{
${ }^{51}$ En este sentido ha sido el discurso del Senador Sherman que se pronunció a favor de su propuesta de Ley, que es destinada a "prevenir y controlar las combinaciones hechas con el propósito de prevenir la competencia o para restringir el Mercado, o para incrementar los beneficios del productor, incrementando, al mismo tiempo, los costes del consumidor"("to prevent and control combinations made with a view to prevent competition or for the restraint of trade or to increase the profits of the producer at the costs of the consumer"- cit. por MORGAN, T.,Modern Antitrust Law and its Origins, op. cit, p.114

${ }^{52}$ MARTÍN LABORDA, A. R. Libre competencia y competencia desleal, ed. La Ley, Madrid, 2001, p. 89.

${ }^{53}$ POSNER, R. Antitrust Law:an Economic Perspective, op. cit., p.vii.

${ }^{54}$ Ver GRAVES, R(ed.) Competition law, Darthmouth Publ., Burlington, 2003.

${ }^{55}$ KOVACIC, W. "Comments and Observations - the Sherman Act", 59 Antitrust L. J. 115,1990.

${ }^{56}$ MAY, J. "The Role of the States in the First Century of the Sherman Act", 59 Antitrust L. J. 93, 1990.

${ }^{57}$ HOVENKAMP, H. "Antitrust Policy, Federalism, and the Theory of the Firm:an Historical Perspective", 59 Antitrust L. J. 75, 1990.

${ }^{58}$ Según SHENEFIELD\&STELZER The Antitrust Laws..., op.cit.,p.12, los conflictos entre objetivos políticos
} 
objetivos y subobjetivos, preestablecida por el Congreso y considero que la tarea de las agencias antitrust es identificar, seguir y aplicar esta jerarquía, en que la idea de dispersión del poder ${ }^{59}$, de equilibrarlo y de prevenir su concentración en un centro determinado ${ }^{60}$, ocupa un nivel principal.

Como se ha enfatizado, el problema de los objetivos en derecho de la competencia es importante para el proceso decisorio de la FTC y la División Antitrust.Se trata de entender cómo funciona el mercado y qué objetivos debe elegir la agencia para su propio funcionamiento.Este problema se relaciona directamente con el estándar ${ }^{61}$, empleado en el proceso decisorio de la FTC y los juzgados-la rule of reason o per se rule ${ }^{62}$.Además, es obvio que para medir la razonabilidad de un acuerdo restrictivo a la competencia de acuerdo con la rule of reason, habrá diferencia si se elige la perspectiva de eficiencia económica, redistribución de la riqueza o dispersión de poder(pudiendo ser asimismo un objetivo "mixto" : por ej.eficiencia y descentralización de poder).A esta misma línea de conclusiones conducen las reflexiones de Joliet, $R^{63}$,según quien para la rule of reason se dan dos interpretaciones

y económicos surgen raras veces en la práctica: la prevención del poder monopolístico contribuye al bienestar del consumidor y responde al objetivo socio-político de dispersar el poder económico.Los dos autores consideran que cuando los objetivos sociales y económicos están en conflicto, prevalecen los objetivos económicos.Considero que las agencias antitrust en su proceso decisorio en la gran parte de los casos buscan un compromiso de objetivos, de acuerdo con una jerarquía determinada. ${ }^{59}$ Ver HOFSTADTER, R. "What Happened to the Antitrust Movement?" en AREEDA\&KAPLOWAntitrust Análisis, ed. Little, Brown and Co., Boston, 1988, pp.54 - 55:En la tesis de HOFSTADTER, analizando la estadística de Eliot, C., algunas corporaciones eran demasiado poderosas y podían dominar los poderes políticos de los estados, y, llegando a acuerdos entre ellas, podrían dominar el gobierno federal.Así se quería detener la concentración de poder para no destruir el gobierno democrático. ${ }^{60}$ Vease en este sentido el discurso del Senador SHERMAN, Congressional Record, $21^{\text {st }}$ Congress, $1^{\text {st }}$ Session, 1890, p.2457;SEPLAKI, L. Antitrust...,op.cit., p.11.

${ }^{61}$ Ver en este sentido AREEDA\&KAPLOWAntitrust Analisis..., op.cit., p.52.

${ }^{62} \mathrm{Se}$ ha de recordadar que la rule of reason ha sido establecida por primera vez en la sentencia Standart Oil Co. of New Jersey v. U. S. 221U. S.1, 1911, donde el Juez White establece la definición de "restricción del comercio" de la Sherman Act.La competencia ha sido declarada objetivo de la Ley para convertirla en un factor regulador del sistema económico.Las interpretaciones tempranas de la Sherman Act dejan en manos del juez la interpretación de "restricción del comercio", de tal modo que la razonabilidad de la conducta debe medir su legalidad, así que es necesario probar no solamente la existencia del acuerdo, sino asimismo sus efectos anticompetitivos.Con el transcurso del tiempo, basándose en su experiencia, los juzgados han decidido que algunos tipos de conductas como fijación de precios(U. S. v. Trans Missoury Freight Assoc.,166 U. S. 290, 1897), división del mercado(U.S. v. Addiston Pipe\& Steel Co., U. S. Court of Appeals, $6^{\text {th }}$ Cir.1898,85 Fed.271), fusiones con efecto anticompetitivo(Northern Securities Co. v. U. S., 193 U. S.197, 1904) , abuso de posición dominante - monopolización (Standart Oil Co. of New Jersey v. U. S. 221 U. S.1, 1911), siempre restringen la competencia y son ilegales "per se".En este caso sólo es necesario probar la existencia del acuerdo y no sus efectos anticompetitivos, aunque la regla "per se" no excluye la posibilidad del acusado de justificar la práctica por aportar beneficios a los consumidores - en esta línea ver GELHORN, E. Antitrust and Economics in a Nuttshell, ed. West Publishing, Minn., 1981, p.25; AREEDA\&KAPLOW Antitrust Analisis, op.cit., p.188 y ss.; HOLMES, W. Antitrust Law Handbook, ed. CDC, Darfeeld, 1994, pp.206- 207, DIRLAM\&KAHN Fair Competition..., op.cit., pp.45-49. 
teóricas contradictorias, según la perspectiva de los objetivos de la agencia antitrust:

De acuerdo con la primera teoría, la rule of reason debe requerir una investigación si las prácticas restrictivas bajo ciertas circunstancias pueden servir al interés público mejor que la libre competencia.En este caso la razonabilidad se relaciona directamente con la racionalidad del acuerdo y con la discrecionalidad de la agencia o el juzgado y se extiende hasta la determinación de los efectos económicos y sociales del acuerdo.Esta doctrina del "interés público" como base de la rule of reason en el derecho antitrust puede convertir la rule of reason en justificación de cualquer restricción de la competencia: nótese, además, que en este caso el "interés público" es un concepto indeterminado y que el análisis debe determinar los intereses de los consumidores y los productores y que un mismo agente de mercado en la mayoría de los casos es consumidor y productor a la vez.

La teoría que ha encontrado más apoyo en la doctrina norteamericana es que la rule of reason participa en el esquema de la construcción de la Sherman Act y es la regla que determina el intento del Congreso de convertir la competencia en base de su política.En este caso la investigación se limita a determinar si una práctica tiene efecto competitivo o no.Aquí la discrecionalidad de la agencia o el juez se extiende hasta determinar la importancia de la restricción. Para Joliet la regla "per se" entra en juego cuando están excluidas todas las justificaciones de la conducta.

Se puede sugerir una tercera visión, muy parecida a la anterior: las agencias cambian su estándar en el proceso decisorio de acuerdo con un cambio en la perspectiva y coordinación de objetivos que se han elegido y este cambio en la perspectiva es indispensable, porque el mercado y la sociedad evolucionan rápidamente y las agencias tienen que adecuar sus métodos a esta evolución ${ }^{64}$.Así, el período de la rule of reason en el derecho antitrust norteamericano(1915-1939) fue seguido por el período de la aplicación del estándar per se(1940-1974) ${ }^{65}$.Durante este período la doctrina es estática:se intenta controlar las prácticas ilegales, evitar prohibiciones de prácticas que pueden ser pro-competitivas y "simplificar" los casos "antitrust" con el riesgo de que esto pueda llevar a una estrechez no deseada en las interpretaciones.En la época moderna del antitrust(entendida a partir del año 1974) se sigue la aplicación del estándar "per se", pero completado por el estándar de razonabilidad(aunque, como se verá a continuación, la doctrina no es unidireccional):se considera que si se introduce únicamente el estándar "per se", esto llevaría a una estrechez en las interpretaciones del derecho antitrust y la aplicación únicamente del estándar de razonabilidad crearía inseguridad para las

\footnotetext{
63 JOLIET, R. The Rule of Reason in Antitrust Law, ed.Martinus Nijnoff, La Haye, 1967, pp.3.4; ver asimismo HANDLER, H. "AStudy of the Construction and Enforcement of the Federal Antitrust Laws", TNECMonogr.38, 1941.

${ }^{64}$ Además, en el H.P.Report 1142, 64d Congress, 2d Session 19, 1914, se puede leer que "es imposible establecer definiciones que puedan enmarcar todas las prácticas anticompetitivas.En este area no existen límites de las invenciones humanas."

${ }^{65}$ Para un análisis más detallado de estos períodos ver MORGAN, T. Modern Antitrust Law and it's Origins, ed. West Publishing, Minn., 1994, pp.33 -800, AREEDA\&KAPLOWAntitrust Analisis.... Op. Cit, pp.200275 y LETWIN, W. Law and Economic Policy..., op. cit, p.96.
} 
empresas.En la sentencia Professional Engineers, 435 U. S. 679(1978) se enfatiza que las dos reglas son complementarias entre si, reemplazando los razonamientos de otra sentencia- Continental T. V. , Inc. v. GTE Sylvania, Inc., 433 U. S. 1(1977), según que la rule of reason prevalece como estándar de análisis en los casos antitrust y "per se" se limita exclusivamente a prácticas que tienen efecto dañiño y no aportan ningun beneficio para la libre competencia ${ }^{66}$.

A continuación considero importante hacer una breve referencia a la regla de la razón flexible que actualmente es base del proceso decisorio de la FTC y los juzgados.

Se considera que los dos estándares contribuyen para evitar la acumulación de errores de las agencias antitrust y los juzgados.Estos errores pueden ser errores positivos(declarar ilegal una práctica lícita) o errores negativos(cuando la conducta ilegal es exculpada). De este modo según algunos autores de la Escuela de Chicago hay que elegir la regla que minimiza la suma de los errores positivos y negativos la rule of reason flexible, minimizando asimismo los costes del proceso decisorio ${ }^{67}$. Además de la minimización de los costes de los errores, la regla de la razón flexible se caracteriza con el ahorro de costes administrativos y judiciales, estableciendo un proceso decisorio flexible y creando, cuando las condicions del caso concreto lo permitan, un modelo simplificado del mercado, tomando en consideración solo los factores relevantes ${ }^{68}$.

Es conveniente hacer, además, algunas notas, también breves, sobre la influencia de las ideas de la Escuela de Chicago y Post - Chicago sobre la perspectiva de los objetivos antitrust, los estándares de decisión y el proceso decisorio de la FTC y los juzgados.

La actividad de la FTC y los juzgados ha sido influida por la Escuela de Chicago en dos aspectos:

En primer lugar, por la creencia de que el único propósito del antitrust es la

\footnotetext{
${ }^{66}$ Un comentario de estos asuntos se puede encontrar en ARTHUR, T. "A Workable Rule of Reason: a Less Ambitious Antitrust Role for tne Federal Courts", 68 Antitrust L. J. 337,2000.

${ }^{67}$ TOM, W. y PAK, C. "Toward a Flexible Rule of Reason", 68 Antitrust L.J.391, 2000;MURRIS, T. "The New Rule of Reason", 57 Antitrust L. J. 858, 1989;BECKNER, C. y SALOP, S. "Decision Theory and Antitrust Rules", 67 Antitrust L. J.41, 1999 y POSNER, R. Economic Analisis of Law, 5ed., ed.Aspen Law and Business, New York, 1998, p.241.

${ }^{68}$ Se dan dos variantes contradictorios: o bien un análisis concreto, considerando todos los factores como en el caso de Chicago Board of Trade v. U. S., 246 U. S. 231, 1918 o conformarse con un modelo simple del mercado, sin considerar las contradicciones de la realidad en todos sus aspectos, porque se considera que el cambio significante de los factores solo afecta el mercado a corto plazo, y, además hay que considerar solo los factores relevantes- SIMON, H. "Administrative Behavior", cit .por TOM, W. y PAK, C. Toward a Flexible..., op.cit., pp.398-399. Algunos autores contemporáneos llegan a la idea del así llamado "quick look" como un instrumento importante del análisis antitrust, cuando se emplea la rule of reason.Se considera que el quick look debe ser empleado solamente a conductas retrictivas de precios que tengan obvios efectos adversos (directos), sin estudiar detalladamente el mercado.En contra de esta visión ver NEESE, A. "Farewell to the Quick Look:redefining the Scope and Content of the Rule of Reason", 67 Antitrust L J., 1999.
} 
maximización del bienestar del consumidor $\left(\mathrm{BORK}^{69}\right)$ y de aquí(o según la mayoría de la doctrina unida a ) la maximización de la eficiencia $\left(\mathrm{POSNER}^{70}\right)$.

En segundo lugar por los intentos de explicar los efectos procompetitivos de prácticas anteriormente aceptadas como anticompetitivas ${ }^{71}$, considerando que los errores positivos de las agencias antitrust entorpecen más el proceso competitivo que los negativos.Se ha afirmado que hay que buscar esquemas y modelos más simples y reglas de legalidad per se de conductas ${ }^{72}$.

En la era de Post-Chicago el objetivo eficiencia no se ha reemplazado, pero se ha intentado superar la búsqueda de esquemas simples, aplicando la teoría de los juegos a varios supuestos de conductas y estableciendo unos modelos de comportamiento del mercado, diferentes de los establecidos por la Escuela de Chicago.Los representantes Post-Chicago consideran que los errores negativos de las agencias tienen más efectos adversos sobre el proceso competitivo que los positivos ${ }^{73}$.

En relación con la elección de objetivos y el proceso decisorio de la FTC se puede resumir, que el análisis jurídico y económico no siempre puede ofrecer una solución única y "justa":es difícil aceptar que en un asunto concreto se puede desglosar toda la actividad económica de una empresa y todas sus relaciones con los consumidores y competidores para determinar las consecuencias a corto y largo plazo.Así, el punto de partida, cuando se empieza a enjuiciar un asunto antitrust tiene que ser el intento de mejorar las condiciones de mercado, considerando adecuadamente los elementos relevantes al caso y los intereses en juego, tomando en consideración los intereses de los consumidores.

A continuación se analizarán los objetivos del derecho antitrúst europeo que coinciden con los objetivos de la Comisión como autoridad encargada con las tareas de defensa de la competencia en Europa y las diferencias que existen con los objetivos de la legislación antitrúst estadounidense.

\section{Los objetivos del derecho de defensa de la competencia europeo y de la Comisión como órgano de defensa de la competencia en Europa.}

Debemos recordar que e el Tratado de la UE tiene como objeto crear un Mercado Común basado en una unión económica plena entre los Estados miembros.Desde esta perspectiva se puede leer el art. 2 TCE, según el cual la Unión tendrá los siguientes objetivos:

- Promover el progreso económico y social y un alto nivel de empleo y

\footnotetext{
${ }^{69}$ BORK, R. The Antitrust Paradox:Policy at War with Itself, ed.Free Press, New York, 1993, p.7.

${ }^{70}$ POSNER, R Antitrust Law:anEconomic Perspective, op.cit., p.4.

${ }^{71}$ TELSER, L. "Why Should Manufacturers Want Free Trade", 3 L. J.\&Ec.86, 1960.

${ }^{72}$ POSNER, R. "The Next Step in the Antitrust Treatment of Restricted Distribution:Per Se Legality", 48 U. Chi. L. Rev.623, 1981 y BORK, R. The Antitrust Paradox, op.cit, p.262.

${ }^{73}$ Ver en general HYLTON, K. y SALINGER, M. "Tying Law and Policy: a Decision-Teoretic Approach", 71Antitrust L. J., 2003.
} 
conseguir un desarrollo equilibrado y sostenible, principalmente mediante la creación de un espacio sin fronteras interiores, el fortalecimiento de la cohesión económica y social y el establecimiento de una unión económica y monetaria que implicará en su momento, una moneda única, conforme a las disposición del presente Tratado,

- Afirmar su identidad en el ámbito internacional, en particular mediante la realización de una política exterior y de seguridad común que incluya la definición progresiva de una política de defensa común que podría conducir a una defensa común, de conformidad con las disposiciones del art. 17,

- Reforzar la protección de los derechos e intereses de los nacionales de sus estados miembros, mediante la creación de una ciudadanía de la Unión,

- Mantener y desarrollar la Unión como un espacio de libertad, seguridad y justicia, en el que esté garantizada la libre circulación de personas conjuntamente con medidas adecuadas respecto al control de las fronteras exteriores, el asilo, la inmigración y la prevención y la lucha contra la delincuencia;

- $\quad$ Mantener el acervo comunitario y desarrollarlo con el fin de examinar la medida en que las políticas y formas de cooperación, establecidas en el presente Tratado deben ser revisadas para asegurar la eficacia de los mecanismos e instituciones comunitarias.

De este modo, existe un amplio alcance de objetivos cuyo fin es la consecución de la integración económica de la Unión Europea: muchas veces el Mercado Común ${ }^{74}$ se ha usado coloquialmente como sinónimo de la Unión Europea con significado de un Mercado donde las barreras de entrada están reducidas y donde las autoridades de los Estados miembros adoptan políticas eficientes para la integración económica, bajo la supervisión de las instituciones de la Unión Europea ${ }^{75}$. Para asegurar el buen funcionamiento de este Mercado resulta indispensable que se haga reinar una competencia justa y libre entre las empresas.En este sentido el art.3, letra c) TCE proclama "el establecimiento de un régimen que garantice que la competencia no será falseada en el mercado común".CRUZ FERRER ${ }^{76}$ en su libro "Principios de la regulación económica en la Unión Europea" analiza las transformaciones económicas después de la Guerra Fría, señalando que los Mercados nacionales van viendo caer las barreras que los protegían de la competencia

\footnotetext{
${ }^{74}$ La importancia de la defensa de la competencia para el Mercado Común está analizada por SOUTY, F. Le droit de la concurrence de l'Union Européene, ed.Montchrestien, Paris, 1997, pp.12 - 14;ver asimismo WAELBROECK, M. y FRIGNANI, A. Derecho europeo de la competencia I, ed. Bosch, Barcelona, pp.7- 14.

${ }^{75}$ Según KORAH, V. Introducción al Derechoy Práctica de la Competencia en la CEE, ed. Ariel, Barcelona 1988, p.11, "en unos mercados geográficamente más extensos podrá haber mayores oportunidades para la especialización. Y en numerosos sectores industriales, las empresas podrán crecer hasta alcanzar tamaños con cuales puedan beneficiarse en producción y distribución, con economías de escala, dejando simultáneamente a otros productores, que puedan competir."

${ }^{76}$ CRUZFERRER, J.Principios de regulación económica en la Unión Europea, ed. Instituto deestudios económicos, Madrid 2002, pp. 37 - 38.
} 
extranjera, integrándose en Mercados transnacionales... que suponen nuevas exigencias de competitividad a las empresas y a los países".

Se ha hecho esta breve introducción para destacar la importancia de la defensa de la competencia para la consecución del objetivo - Mercado Común, considerando asimismo los demás objetivos del derecho y de las autoridades de defensa de la competencia como la eficiencia, el bienestar del consumidor, etc... ${ }^{77}$.El objetivo Mercado Común es de especial importancia y más cuando se trata de los procesos actuales de integración y ampliación de la Unión Europe ${ }^{78}$ Así, a través de la política de la competencia se define el interés económico común de la Unión Europea $^{79}$ a la luz de la Estrategia de Lisboa, porque la UE se ha puesto unos propósitos ambiciosos- en 10 años conseguir ser la economía más competitiva y dinámica del mundo ${ }^{80}$.

En este orden de razonamientos y con propósito de establecer un equilibrio

${ }^{77}$ Véase VAN MIERT, K. Foreword en EEKHOFF, J.(ed.) Competition Policy in Europe, ed.Springer, Berlin, 2004, p.V y MOCH, C. "Competition- the Core of a Market Economy" en el mismo libro de EEKNOFF; SLOT, P. ""A View from the Mauntain: 40 Years of Development in EC Competition Law", CMLR, vol.41/2, abril, 2004; BELLAMY, C. y CHILD, G. Derecho de la competencia en el Mercado Común, ed. Civitas, Madrid, 1992, p.38; GIL IDAÑEZ, J.L. "La Comisión y la aplicación del derecho comunitario de la competencia" en GARRDO ESPÁ, L. La defensa dela competencia por losórganosjudiciales: el Reglamento CEN 1/ 2003, ed. Consejo General del Poder Judicial, Madrid, 2005, p.112. - las libertades políticas-la competencia efectiva-la política cultural, medioambiental y social comunitarias-los objetivos del derecho nacional-últimamente se enfatiza en la protección de los derechos de los consumidores y usuarios como objetivo del Derecho Europeo de la Competencia ${ }^{78}$ Según AMATO, G. Antitrust and theBounds of Power: TheDilemma ofLiberal Democracy in the Historyof the Market, ed. Hart Publishing, Oxford 1997, p.44, la política de competencia está subordinada a la integración de los mercados; en este sentido SOUTY, F. Le droit de la concurrente", op. cit., pp.17-20 y recientemente PIETOVIC, K. "Reform of EC Antitrust Enforcement: Criticism of the New Sistem is Highly Exaggereted", ECLR N 25 - 6/ 2004, p.356; ver asimismo KORAH, V. Introducción al Derecho..., op.cit. p.19 y An Introductory Guide to EC Law and Practice, 8va edición, ed.Hart Publishing, Oxford 2004, p.2, 6 , 7 y 12 citando el asunto 56 y 58/64 Consten \& Grundig c. Comisión(1966) Rep.299 y analizando los objetivos del Derecho Europeo de la Competencia, distintos del objetivo - eficiencia(EEUU):

- la integración de los mercados

- la competencia "justa"

- los beneficios para la pequeña y mediana empresa

- las libertades políticas

- la competencia efectiva

- la política cultural, medioambiental y social comunitarias

- los objetivos del derecho nacional

- últimamente se enfatiza en la protección de los derechos de los consumidores y usuarios como objetivo del Derecho Europeo de la Competencia

${ }^{79}$ EEKHOFF, J. "Preface" en EEKHOFF , J.(dir.) Competition Policy in Europe, ed.Springer, Berlin, 2004, p.VII.

${ }^{80}$ En relación con la política de competencia y la Estrategia de Lisboa véase KROES, N. "European Competition Policy- Delivering Better Markets and Better Choices", discurso de 15 de septiembre de 2005, disponible en www.europa.eu.int/comm/speeches ; En palabras de MONTI, M. "en primer lugar, la política de competencia debe contribuir al objetivo de Lisboa de hacer de Europa la economía de conocimiento más dinámica del mundo" en Prólogo al "XXXIII Informe sobre la política de la competencia", ed. Comisión Europea, Luxemburgo, 2003, p.3. 
económico, social y político en el marco comunitario, ¿cual de los objetivos de la Comisión debe prevalecer: la protección del Mercado único o la eficiencia económica, sugiriendo una jerarquía de objetivos, organizada con base el principio de distribución de poder económico( y político) a escala global?

En este sentido WAELBROECK y FRIGNANI" afirman que "en el marco comunitario, la tensión entre estos imperativos encontrados nunca ha sido completamente resuelta...".Creo que, en el momento actual, la preferencia es clara respecto al Mercado único y coincido con GARRIDO ESPÁ que afirma: "En el seno de la Unión Europea, la política de competencia debe añadir a tales designios el más específico de reforzar la integración y unidad del Mercado interior(el Mercado Común), velando por la eliminación de conductas que tiendan a compartimentar artificialmente el Mercado único o a restringir el comercio entre los Estados- miembros, cuidando asimismo de evitar que éstos concedan un tratamiento preferencial a las empresas nacionales" 82 .Es conveniente considerar asimismo la afirmación de los autores anteriormente citados $^{83}$, que "el enfrentamiento de los objetivos de integración y de eficacia no es absoluto: a largo plazo la integración no puede sino beneficiar a la eficacia del proceso económico".En esta misma línea cabe observar lo siguiente: en la doctrina es comunmente aceptado que los objetivos del derecho antitrust estadounidense $\mathrm{e}^{84} \mathrm{y}$ del derecho de defensa de la competencia comunitario(y de las autoridades de defensa de la competencia) difieren parcialmente, porque los EE.UU son una sola nación, con un mercado único plenamente operativo, a diferencia de Europa, donde ha habido que construir, y hay que seguir construyendo este Mercado por encima de las barreras nacionales ${ }^{85}$.En este sentido DIEZ ESTELLA hace referencia a las siguientes cuestiones que hacen diferir las políticas estadounidense y europea en materia de defensa de la competencia ${ }^{86}$ en relación con sus objetivos:

- la idea de integración de los mercados no existe en el derecho antitrúst norteamericano

mientras que desde los años -70 en EEUU la eficiencia asignativa ha sido uno de los principios rectores de la mayoría de las decisiones de política legislativa y judicial en EEUU, en Europa se da una mayor importancia a consideraciones de caràcter social y político

- $\quad$ mientras que en EEUU el sistema de aplicación del derecho antitrúst es

${ }^{81}$ WAELBROECK, M. y FRIGNANI, A. Derecho europeo de la competencia “, ed.Bosch, Barcelona, 1998, p.24. 82 GARRIDO ESPÁ, L. "Los tribunales del orden civil y el Reglamento(CE) N 1/ 2003 del Consejo de 16 de Diciembre de 2002, relativo a la aplicación de las normas sobre competencia previstas en los artículos 81 y 82 del Tratado" en GARRIDO ESPÁ, L.La defensa de la competencia por los órganos judiciales: el Reglamento(CE) N 1/ 2003, ed.Consejo General del Poder Judicial, Madrid, 2005, p.46.

${ }^{83}$ WAELBROECK, M. y FRIGNANI , A. Derecho europeo de la competencia I, op.cit., p.24.

${ }^{84}$ Véase pp.15- 18, supra.

${ }^{85}$ VELASCO SAN PEDRO, L.A. "El derecho europeo de la competencia" en VELASCO SAN PEDRO , L.A. Derecho europeo de la competencia:antitrust e intervenciones públicas, ed.LexNova, Valladolid, 2005, p.41.

${ }^{86}$ Ver DIEZ ESTELLA, F. "Los objetivos del derecho antitrúst", GJUEC, N 224, marzo/abril 2003, pp.32-52. 
eminentemente descentralizado, en la UE las potestades más importantes se atribuyen a la Comisión(y, rigiendo los principios de proporcionalidad y subsidiariedad, a la Red de Autoridades Europeas de Competencia)

- mientras en EEUU el tamaño de la empresa no era un objetivo a coseguir, en la UE sí se quiería estar en situación de competir eficientemente con las grandes corporaciones transnacionales: por esta razón en Europa no se prohiben las posiciones de dominio, sino solo el abuso de las mismas

- $\quad$ en la UE existe una mayor confianza en que la intervención de los gobiernos pueda solucionar los problemas existentes en el mercado

En mi opinión, además, a través de la defensa de la competencia y a través de las demás políticas comunitarias, llevadas a cabo por las autoridades comunitarias, se realiza una distribución de poder económico(y político) a escala regional(en el ámbito de Europa) como un paso en dirección al establecimiento de un equilibrio a escala global.En este sentido, la Comisión ocupa un lugar primordial, siempre insistiendo en la complejidad de los procesos de integración y ampliación de la Unión Europea.

Se puede afirmar que la integración de los mercados en el ámbito europeo forma parte del proceso de expansión de los mercados a escala global como una manifestación de la globalización económica.En mi opinión, más que todo, estos procesos de ampliación de los mercados globales, basado su funcionamiento en el principio de libre competencia, han impulsado la creación, la integración y la ampliación de la Unión Europea, basándose en las ideas liberales.En este contexto, desde una perspectiva darwiniana M.Sanson- Hermitte afirma que "la idea motriz es la automaticidad: si se suprimen todas las barreras entre los países europeos, el resto se efectuará por un encadenamiento de causas y de efectos que conducen a la mejor de las sociedades posibles" ${ }^{17}$.Cabe preguntarse $¿$ cómo sería la mejor sociedad posible?Comparto la visión de SOROS $^{88}$ de que el mercado es "amoral" y creo que para conseguir una mejor sociedad, lejos de las utopías, que existen desde tiempos remotos, es necesario el establecimiento de un equilibrio económico, social y político.Quizá el hombre ha progresado en sus inventos y descubrimientos, hasta el momento, únicamente imitando la naturaleza. Y el equilibrio natural, basado en varios desequilibrios y contraposiciones de diferente índole o su continuo proceso de establecimiento son las más evidentes manifestaciones de la misma.Quisiera tener razón al afirmar que los autores de los Tratados han tenido alguna intención similar al respecto, estableciendo un sistema de valores económico- políticos, pero asimismo sociales y convirtiendo dicha unión regional- la Unión Europea- en un modelo de tal sociedad, basada en el equilibrio económico, social y político o en un continuo establecimiento de equilibrios a nuevos niveles que se refuerzan por el derecho de

\footnotetext{
${ }^{87}$ Cit. por CALVO CARAVACA, A.L. y CARRASCOSA GONZÁLEZ, J. "El derecho europeo de la competencia: objeto, fuentes y sistemática" en CALVO CARAVACA, A.L. y MORALES LIMONES ,P.B. Derecho europeo de la competencia, ed.Colex, Madrid, 2000, p.166.

${ }^{88}$ SOROS, G. La crisis del capitalismo global, ed.Debate, Madrid, 1998, p.240.
} 
defensa de la competencia europeo.

A modo de conclusión resumiría que para entender los objetivos actuales de las autoridades antitrust norteamericanas y europeas se deben buscar las causas de la creación de dichas autoridades, enfatizando las diferentes condiciones económicas, políticas y sociales en los dos lados del Atlántico.En el derecho estadounidense se ha sostenido unánimamente que las políticas de defensa de la competencia y de defensa de los consumidores y usuarios tienen una finalidad: la maximización de la eficiencia que lleva a la maximización del bienestar del consumidor, pero también se han propuesto otras visiones respecto a los objetivos de estas autoridades.Una de ellas es que el Congreso no ha perseguido un único objetivo, creando leyes y autoridades antitrust, sino que su intención ha sido "multidimensional", es decir no existe un objetivo dominante, sino una jerarquía de objetivos y subobjetivos, preestablecidos por el Congreso, en la que la idea de dispersión del poder económico, de equilibrarlo y de prevenir su concentración en un centro determinado ocupa un nivel principal.Para algunos se trata de un mecanismo de conservar el sistema existente antes de la industrialización, suprimiendo centros de poder económico excesivo que amenazan con romper el orden democrático.

Respecto a los objetivos del derecho antitrúst europeo y de la Comisión Europea en la aplicación de la política de competencia se deben recordar los objetivos de los Tratados, donde la idea clave es la creación e integración del Mecado Unico.Este objetivo no excluye otros de índole económica, social y política.Además, a largo plazo se puede producir una covergencia entre los objetivos del derecho europeo y las finalidades del derecho antitrúst estadounidense, estableciéndose una jerarquía de objetivos en aras de la globalización económica y el posible establecimiento de una regulación global de la defensa de la competencia. 
